\title{
Anti-CD19-CD28-zeta modified CAR CD3+ T Lymphocytes JCAR015
}

National Cancer Institute

\section{Source}

National Cancer Institute. Anti-CD19-CD28-zeta modified CAR CD3+T Lymphocytes

JCAR015. NCI Thesaurus. Code C133718.

Genetically modified CD3-positive-enriched autologous T-lymphocytes transduced with a replication incompetent gamma retroviral vector expressing a chimeric T-cell antigen receptor (CAR) consisting of an anti-CD19 single chain variable fragment (scFv), fused to the extracellular, transmembrane and intracellular signaling domains of the T-cell costimulatory receptor CD28 and the cytoplasmic signaling domain of the zeta chain of the TCR/CD3 complex (CD3-zeta) (CAR19-28z), with potential antineoplastic activities. Upon intravenous administration, autologous CD19-28z CAR-expressing CD3+ T-lymphocytes are directed to CD19-expressing tumor cells, and, upon binding to the T-cells, induce selective toxicity in CD19-expressing tumor cells. CD19 antigen is a B-cell specific cell surface antigen expressed in all B-cell lineage malignancies. The CD28 co-stimulatory molecule signaling domain enhances activation and signaling after recognition of CD19. The inclusion of the CD28 signaling domain may increase proliferation of T-cells and antitumor activity compared to the inclusion of the CD3-zeta chain alone. 